\title{
Internal Carotid Artery Dissection with Traumatic Pseudoaneurysm Formation after Penetrating Head Injury
}

\author{
Jeong Sik Ham ${ }^{1}$, Jang Hun Kim ${ }^{1,2,3}$, Won-Ki Yoon ${ }^{3}$ \\ ${ }^{1}$ Department of Neurosurgery, The Armed Forces Capital Hospital, Seongnam, Korea \\ ${ }^{2}$ Trauma Center, The Armed Forces Capital Hospital, Seongnam, Korea \\ ${ }^{3}$ Department of Neurosurgery, Korea University Guro Hospital, Seoul, Korea
}

\author{
Received: July 29, 2019 \\ Accepted: August 8, 2019 \\ Corresponding Author: \\ Jang Hun Kim, M.D. \\ Trauma Center, The Armed \\ Forces Capital Hospital 81 \\ Saemaeul-ro, 177 beon-gil, \\ Bundang-gu, Seongnam-si, \\ Gyeonggi-do 13574 Korea \\ Tel: +82-10-3607-6745 \\ E-mail: jhkimns@naver.com
}

\begin{abstract}
Traumatic pseudoaneurysm of the intracranial arteries is a devastating event leading to high mortality. Its prevalence is rare, and associated with blunt or penetrating head injuries. Here, we describe a case of a 46-year-old woman who was transferred to our hospital following a stab-induced head trauma. Owing to multiple assaults by a sharp knife, the patient presented with an injured intracranial internal carotid artery that formed dissecting pseudoaneurysms. Stupor mentality was noted, and brain computed tomography revealed traumatic subarachnoid hemorrhage, and intracerebral hemorrhage in a "knife shape". Diagnostic angiography showed an active extravasation in the middle cerebral artery and distal internal carotid artery with two pseudoaneurysms. Immediate trapping was planned, and embolization was performed with detachable coils. The patient was transferred to the operation room and emergent decompressive craniectomy with wide durotomy was performed. Unfortunately, the patient expired few days after the operation despite maximal neurocritical care.
\end{abstract}

Keywords: Internal carotid artery dissection; Penetrating head injury; Pseudoaneurysm

\section{INTRODUCTION}

Trauma-related pseudoaneurysms of intracranial arteries are extremely rare, and are known to be associated with blunt or penetrating head injuries ${ }^{2}$. The reported prevalence of traumatic cerebrovascular injury is $0.8 \%$ among all traumatic brain injury (TBI) patients ${ }^{10)}$. The clinical presentation shows a wide range of signs and symptoms, including headache, loss of consciousness, altered mentality, and subarachnoid hemorrhage (SAH) in brain computed tomography (CT), and such neurological deficits may appear either immediately, or months to years later ${ }^{1)}$. Due to the aforementioned reasons, surgeons sometimes fail to identify vas- cular injury in TBI patients, which can lead to devastating results. Therefore, in a case of TBI with an accompanying vascular injury, care and attention is needed to avoid poor prognosis.

Here, we describe a rare case of a woman presenting with a stab-induced penetrating injury to her cranium; she was assaulted by a sharp knife that directly injured her intracranial internal carotid artery (ICA) and led to the formation of dissecting pseudoaneurysms.

\section{CASE REPORT}

A 46-year-old female visited emergency center (Korea Univer-

Copyright (C) 2019 The Korean Neurointensive Care Society

This is an Open Access article distributed under the terms of the Creative Commons Attribution Non-Commercial License (http://creativecommons.org/licenses/by-nc/4.0/) which permits unrestricted non-commercial use, distribution, and reproduction in any medium, provided the original work is properly cited. 
sity Guro Hospital) after a stab-induced head trauma. According to eyewitness evidence, one man diagnosed with schizophrenia stabbed the patient with a knife several times. On admission, she was in a stupor and had seven stab wounds on her face, head, and neck (Fig. 1). Initial Glasgow coma scale (GCS) was 6 (E1V1M4).

She was intubated and her facial bleeding was controlled with simple sutures and compression. Subsequent brain CT revealed traumatic intracerebral hemorrhage (ICH) and SAH mainly on the left hemisphere. Comminuted skull fracture was shown in a temporal lesion and cerebral artery injury was suspected (Fig. 2). We noted a chance of the knife piercing the temporal hemisphere and injuring the circle of Willis.

In our hospital, the interventional suite is adjacent to the emer-

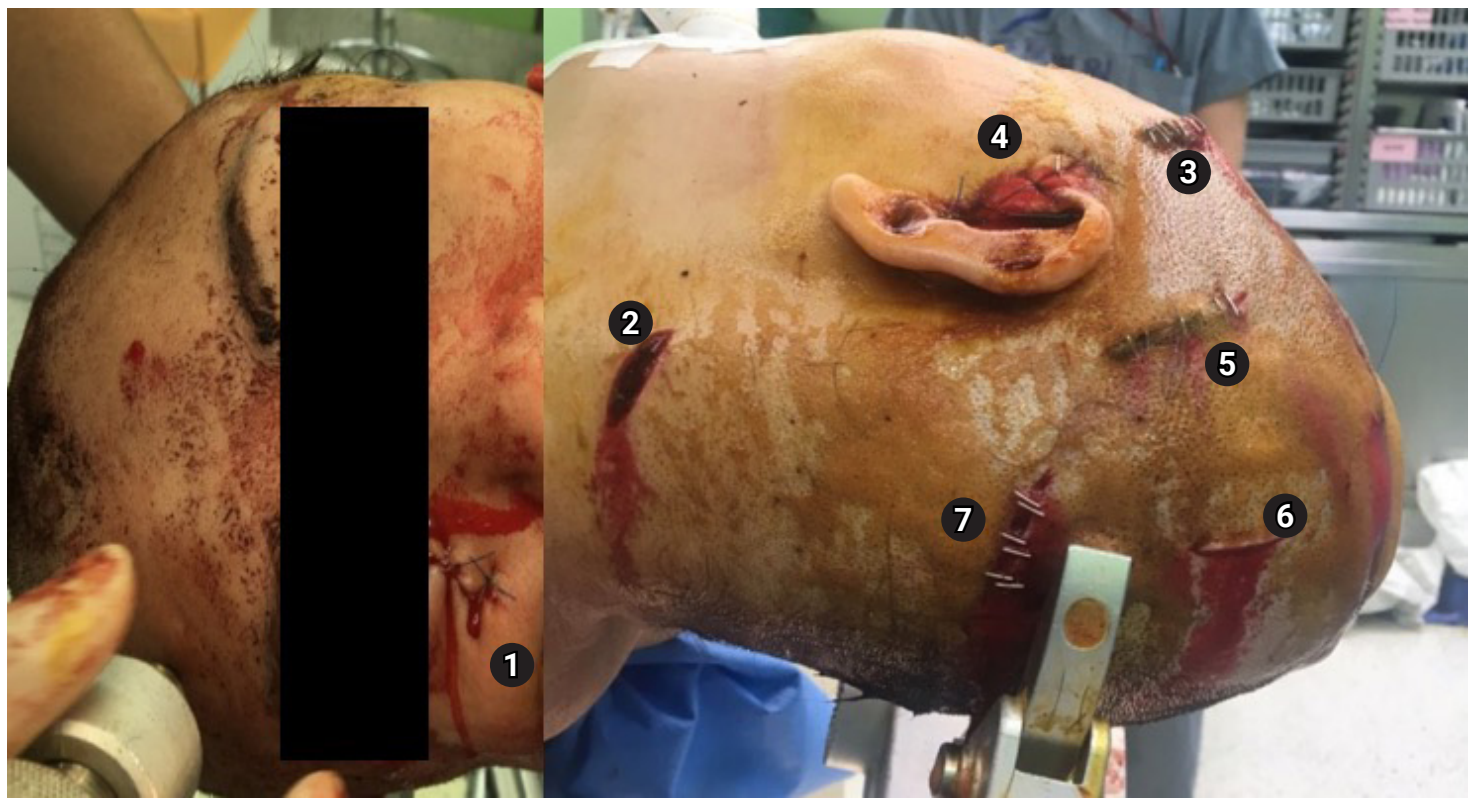

Fig. 1. Seven stab injuries are noted: (1) right lower eyelid, (2) left posterior triangle of lateral neck, (3) frontal, (4) lower temporal, (5) upper temporal, (6) parietal, and (7) occipital area of head, respectively.

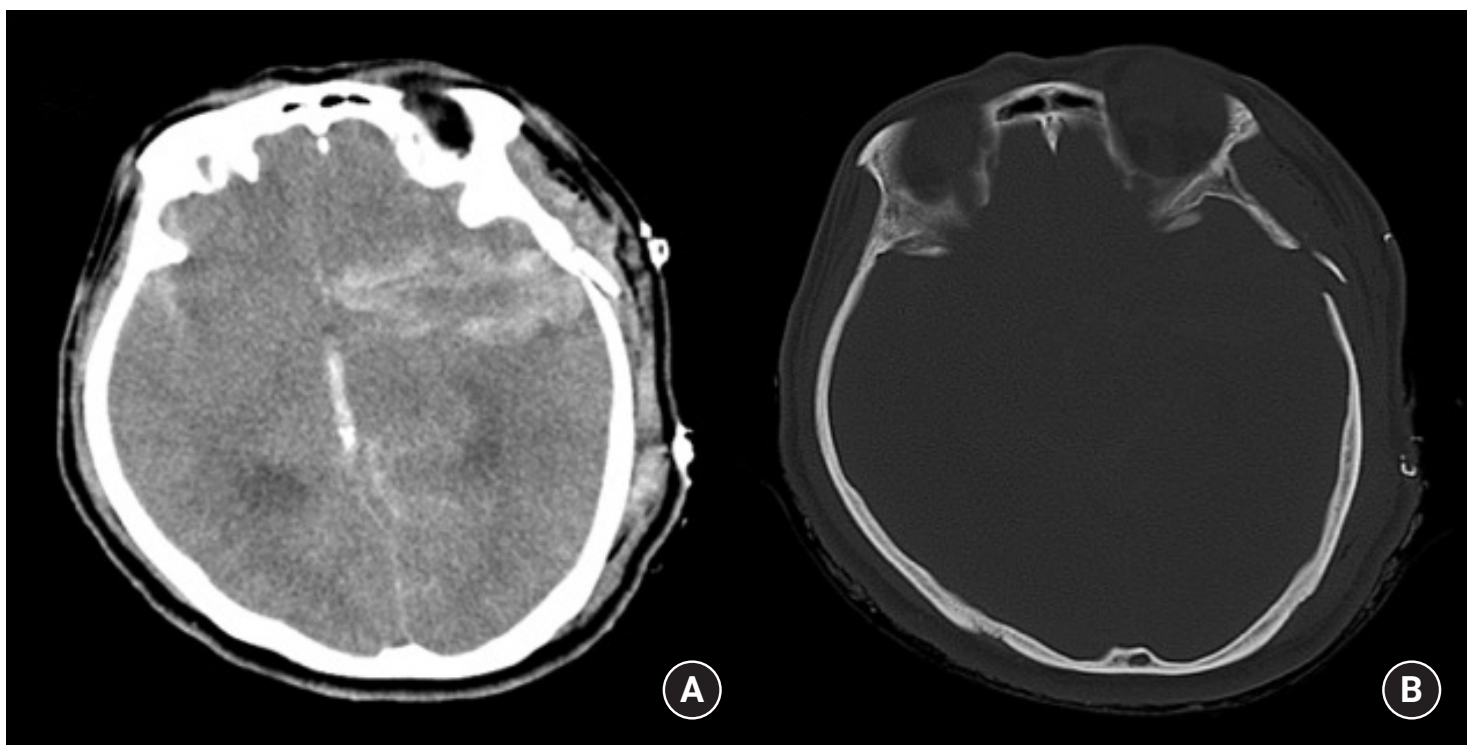

Fig. 2. Initial brain CT reveals left temporal bone fracture, SAH on both sylvian fissures, IVH in the third ventricle, and traumatic "knife-shaped" ICH in the left hemisphere (A). Bone setting of the brain CT presents a comminuted skull fracture of the left temporal bone of the size of the knife (B).

$\mathrm{CT}$, computed tomography; SAH, subarachnoid hemorrhage; IVH, intraventricular hemorrhage; ICH, intracerebral hematoma. 
gency center and she was transferred to the interventional suite immediately. The femoral artery was punctured within $10 \mathrm{~min}$ utes after CT evaluation.

First, diagnostic angiography was performed (Fig. 3). A 7 Fr sheath and 5 Fr guide catheter (Glidecath Headhunter 1; Terumo, Somerset, NJ) was inserted into the right ICA. Angiograms revealed collateral blood flow running from the right to left via the anterior communicating artery. With left common carotid artery (CCA) angiography, the left ICA was not observed. ICA dissection and occlusion was suspected.

After diagnostic angiography, we inserted an intermediate catheter (Envoy Distal Access; DePuy Synthes, Raynham, MA) into the left ICA and attempted angiography again. Angiograms showed an active extravasation in the left first segment of the middle cerebral artery (MCA) and distal ICA. Multiple pseudoaneurysms were noted.

Considering that an emergent decompressive craniectomy should be performed due to malignant brain swelling with increased intracranial pressure (IICP), immediate trapping of left ICA was planned. A microcatheter (Prowler Select Plus; Codman, Raynham, MA) with a microwire (Synchro-2; Stryker, Fremont, CA) was advanced to the left distal ICA. In total, 6 EA detachable coils were embolized. We also added 33\% cyanoacrylate glue for additional hemostasis to the embolized coils. On the final angio-
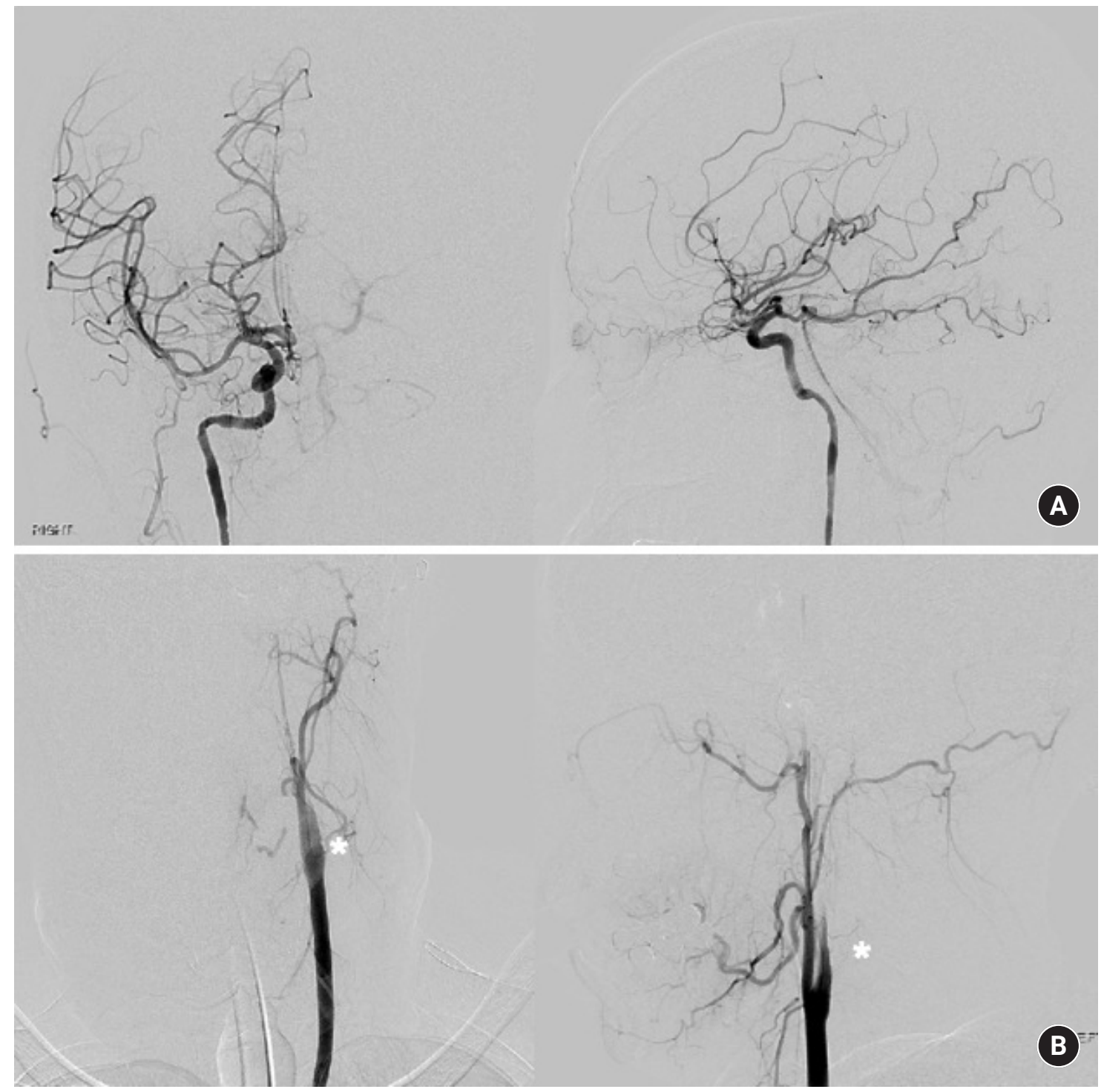

Fig. 3. Right ICA angiogram reveals normal vasculature of the right intracerebral arteries. Collateral blood flows from the right to left hemisphere through the A-comm artery (A). Left CCA angiogram shows preservation of all the ECA branches except STA. However, the ICA is not visible on the proximal portion and we suspect ICA injury somewhere in the distal part (B).

ICA, internal carotid artery; A-comm, anterior communicating; CCA, common carotid artery; STA, superficial temporal artery. 


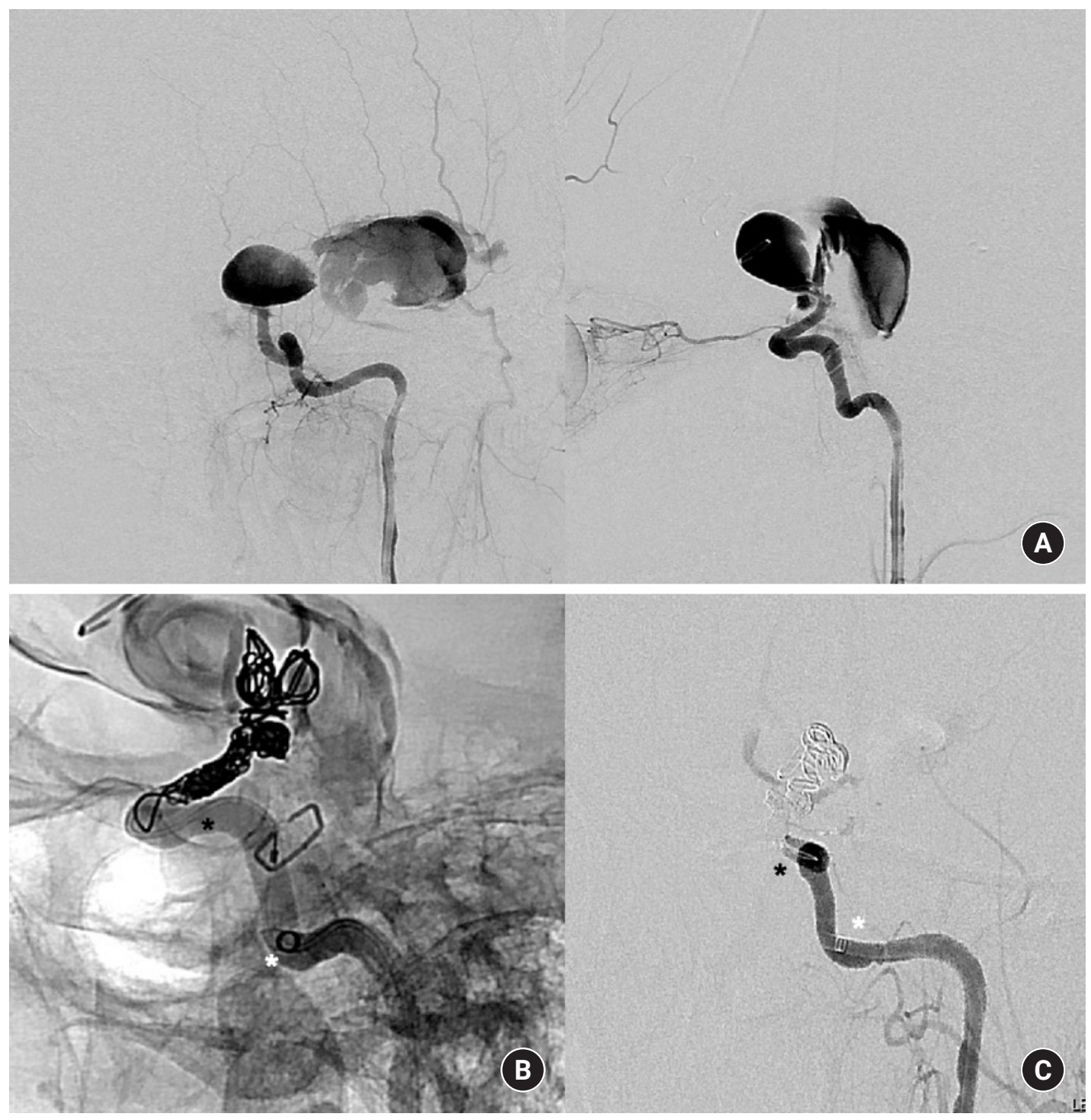

Fig. 4. Left ICA angiogram notes that two large pseudoaneurysms were formed in the anterior and lateral directions (A). An intermediate catheter was inserted into the left ICA and a microcatheter was navigated to the traumatic pseudoaneurysm. Six coils were embolized in the pseudoaneurysm as well as in the distal ICA (B). During the final angiography, no leakage of contrast was noted (C).

ICA, internal carotid artery.

gram, no evidence of acute extravasation was noted (Fig. 4). The whole procedural time (puncture to closure) was 48 minutes.

She was transferred to the operation room and general anesthesia was induced. Emergent decompressive craniectomy with wide durotomy was performed. However, the skin incision was made 40 minutes after closing the femoral puncture site. In operation findings, severe brain swelling was observed and intraparenchymal hematoma was noted. Wide craniectomy with ICH removal was rapidly carried out. Duroplasty with artificial dura was followed and closure of skin was performed after the closed suction system was placed (Fig. 5). Postoperatively, she was comatose, and both her pupils were dilated and fixed. Despite intensive neu- rocritical care, she expired 3 days after operation.

\section{DISCUSSION}

Penetrating injuries to the cranium are devastating events which result in high rates of mortality and morbidity ${ }^{5}$. Initial GCS score and head trauma severity are important prognostic factors ${ }^{4}$. When a patient experiences IICP, emergent decompression is readily necessary. Moreover, if a cerebral arterial injury was accompanied, it would be a greater challenge for the surgeon to manage it. A hasty craniotomy without managing vessel injury might worsen the situation, and therefore, an integrated approach combining surgical 


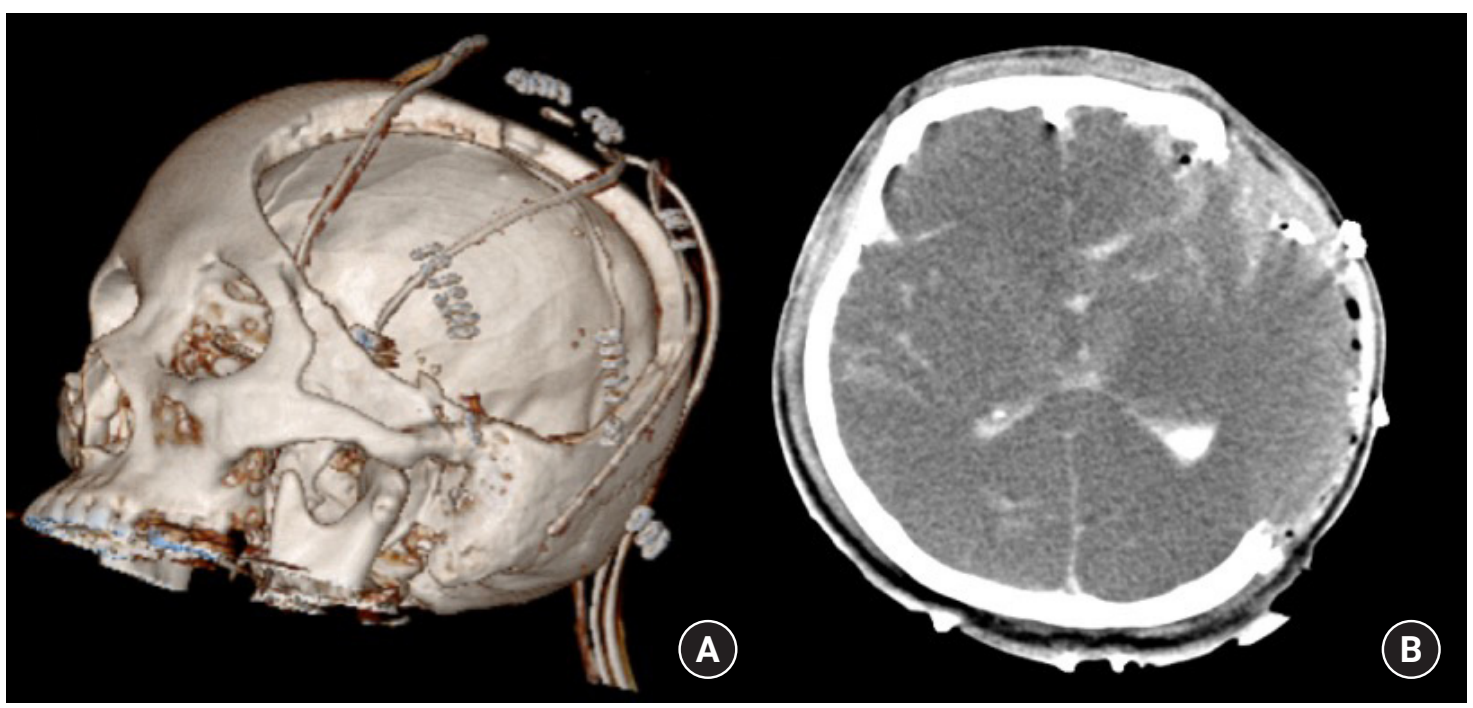

Fig. 5. Decompressive craniectomy with wide durotomy was followed (A). Postoperative brain CT reveals a black brain with severe brain swellings (B).

and endovascular treatment is needed, particularly in cases of ICA dissection with pseudoaneurysm, as in this report.

A majority of the traumatic intracranial aneurysms have been reported as a result of direct vascular injury secondary to penetrating injury or comminuted skull fracture ${ }^{7,8)}$. Blunt-trauma-related pseudoaneurysms rarely form as a result of shearing forces causing torsion and overstretching of the arteries at the time of trauma ${ }^{7,8)}$, though the frequency of the cause of traumatic aneurysms may depend on local factors ${ }^{3)}$.

In this case, the direct penetrating injury dissected the distal ICA and tore the vessel lumen. Histologically, trauma-related aneurysms have been categorized as true, false, and dissecting aneurysms $^{2}$. Among these types, false aneurysms resulting from the destruction of three layers of the vessel wall are induced primarily by head trauma. Traumatic "pseudo- or false aneurysms" are consequently formed, which are thought to be sensitive to rupture compared to true aneurysms.

Traumatic aneurysms should be suspected in cases of acute neurological deterioration following a penetrating head injury, and patients should undergo immediate CT scanning ${ }^{9)}$. If the scan demonstrates SAH in a pattern suggestive of aneurysmal SAH, an angiogram should follow to identify any vascular injury ${ }^{7,8)}$. In this case, the CT feature was quite different from that of usual aneurysmal SAH. Comminuted skull fracture of temporal bone was observed, and ICH was presented at the skull base in a "knife shape" with accompanying SAH. At the tip of the knife, the circle of Willis was located and we could easily identify the intracranial vascular injury. As expected, distal ICA was dissected, and immediate trapping was followed.
Unfortunately, decompressive craniectomy was delayed almost two hours because of vascular interventional therapies, and the transfer from the interventional suite to the operation room. In terms of neurointensive care, a hybrid operation setting might reduce delays ${ }^{6}$; in this case, the delays might have worsened the patient's neurological status. Therefore, we believe that interdisciplinary approaches and hybrid preparation would have otherwise, led to the patient's survival, and are recommended in such cases of TBI with accompanying vascular injuries.

\section{CONCLUSION}

Here, we describe a rare case of a woman presenting with a stab-induced penetrating injury to her cranium; she was assaulted by a sharp knife that directly injured her intracranial ICA and led to the formation of dissecting pseudoaneurysms. Left ICA trapping with coil embolization and followed decompressive craniectomy was immediately performed, however, the patient was expired few days later.

\section{NOTES}

\section{Conflict of interest}

No potential conflict of interest relevant to this article was reported.

\section{ACKNOWLEDGEMENTS}

None. 


\section{REFERENCES}

1. Asari S, Nakamura S, Yamada O, Beck H, Sugatani H. Traumatic aneurysm of peripheral cerebral arteries. Report of two cases. J Neurosurg 1977;46:795-803.

2. Burton C, Velasco F, Dorman J. Traumatic aneurysm of a peripheral cerebral artery. Review and case report. J Neurosurg 1968;28:468-474.

3. Cohen JE, Gomori JM, Segal R, Spivak A, Margolin E, Sviri G, et al. Results of endovascular treatment of traumatic intracranial aneurysms. Neurosurgery 2008;63:476-485; discussion 485476.

4. Gutierrez-Gonzalez R, Boto GR, Rivero-Garvia M, Perez-Zamarron A, Gomez G. Penetrating brain injury by drill bit. Clin Neurol Neurosurg 2008;110:207-210.

5. Kazim SF, Shamim MS, Tahir MZ, Enam SA, Waheed S. Management of penetrating brain injury. J Emerg Trauma Shock 2011;4:395-402.
6. Kinoshita T, Hayashi M, Yamakawa K, Watanabe A, Yoshimura J, Hamasaki T, et al. Effect of the Hybrid Emergency Room System on Functional Outcome in Patients with Severe Traumatic Brain Injury. World Neurosurg 2018;118:e792-e799.

7. Nishioka T, Maeda Y, Tomogane Y, Nakano A, Arita N. Unexpected delayed rupture of the vertebral-posterior inferior cerebellar artery aneurysms following closed head injury. Acta Neurochir (Wien) 2002;144:839-845; discussion 845.

8. Paiva WS, Andrade AF, Sterman Neto H, de Amorim RL, Caldas JG, Teixeira MJ. Traumatic pseudoaneurysm of the superior cerebellar artery. J Trauma Acute Care Surg 2012;72:E115.

9. Schwartz RB, Tice HM, Hooten SM, Hsu L, Stieg PE. Evaluation of cerebral aneurysms with helical CT: correlation with conventional angiography and MR angiography. Radiology 1994;192:717-722.

10. Tunthanathip T, Phuenpathom N, Saehaeng S, Oearsakul T, Sakarunchai I, Kaewborisutsakul A. Traumatic cerebrovascular injury: Prevalence and risk factors. Am J Emerg Med 2019. 